\title{
LDPC and Turbo Coding Assisted Space-Time Block Coded OFDM
}

\author{
M. Y. Alias, F. Guo, S. X. Ng, T. H. Liew, ${ }^{1}$ L. Hanzo
}

Dept. of ECS., Univ. of Southampton, SO17 1BJ, UK.

Tel: +44-703-593 125, Fax: +44-703-593045

Email: ${ }^{1}$ lh@ecs.soton.ac.uk, http://www-mobile.ecs.soton.ac.uk

\begin{abstract}
Space-time block coded OFDM is capable of achieving substantial diversity gains, while supporting high bit-rates in wireless communications. By concatenating a space-time block coded OFDM scheme with powerful channel codes, the performance of the system can be further enhanced. In this contribution both Low-Density Parity-Check (LDPC) coding and turbo coding assisted $G_{2}$ space-time block coded OFDM is investigated. The achievable performance is studied as a function of the number of iterations, coding delay, code rate and decoding complexity.
\end{abstract}

\section{INTRODUCTION}

The combination of space-time coding and orthogonal frequency division multiplexing (OFDM) [1] results in an enhanced system performance in wideband wireless channels $[2,3]$. For the sake of further improving the performance, Forward-Error Correction (FEC) schemes such as Turbo Codes (TC) and Reed-Solomon codes may be invoked for protecting the subcarriers against frequency-selective fading in an OFDM environment.

In recent years, the family of Low Density Parity Check (LDPC) codes has re-emerged as an attractive alternative to turbo coding [3]. LDPC codes were originally proposed by Gallager [4] in 1962. Owing to the codes' capability of approaching Shannon's performance limits, LDPC codes have been applied in conjunction with BPSK for transmission over both AWGN and frequency selective fading channets in OFDM systems [5]. It has also been shown in [6] that LDPCbased space-time coded OFDM systems are capable of efficiently exploiting the achievable spatial diversity in wireless channels.

In this contribution, we comparatively study TC and LDPC aided space-time block coded [7] OFDM. We evaluate the attainable Bit Error Rate (BER) and Frame Error Rate (FER) performance when communicating over 2-path Rayleigh fading channels having a total delay-spread of $5 \mu s$ and quantify the achievable coding gain versus complexity upon varying the number of iterations and code- lengths as well as the BER and FER for various coding rates.

\section{SYSTEM MODEL}

The architecture of our system is shown in Figure 1. At the transmitter, the information source generates random information data bits. The information bits are then encoded by the TC or LDPC encoder. We employed the $\mathrm{TC}(2,1,4)$ code $[3]$ using the parameters shown in Table 1 . The mapping of the data bits and parity bits of the TC encoder to the various modulation constellations was carried out such that it yielded the best achievable performance along with the application of the random separation channel interleaver [3].

We invoked an $(N, K)$ LDPC code defined by the $(M \times N)$ dimensional parity-check matrix [4], where $K=(N-M)$ is the uncoded-information block length. LDPC codes belong to the family

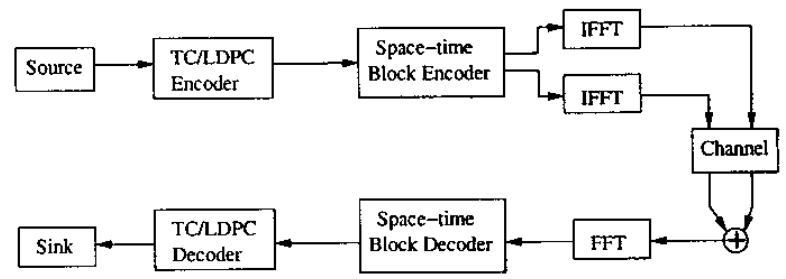

Figure 1: System overview of one-receiver $\mathbf{G}_{2}$ space-time block coded OFDM.

\begin{tabular}{|l|r|}
\hline Code & $\mathrm{TC}(2,1,4)$ \\
\hline $\begin{array}{l}\text { Octal Generator } \\
\text { polynomial }\end{array}$ & 13,15 \\
\hline No. of trellis states & 8 \\
\hline Decoding algorithm & Log-MAP \\
\hline
\end{tabular}

Table 1: Parameters of the $\mathrm{TC}(2,1,4)$ codec.

of linear block codes. These codes are defined as codes using a sparse parity-check matrix having the same number of is per column (column weight, $k$ ) and the same number of Is per row (row weight, $j$ ), where both of these numbers are small compared to the block length $N$. In our simulations we chose $k$ and $j$ values of 3 . The code rate, $R$, of the LDPC codes is given by $R=K / N$. The appropriate values of $K$ and $M$ can then be calculated for the different values of $N$ and $R$.

An example of the parity check matrix is given by:

$$
\mathbf{C}=\left[\begin{array}{llllllllll}
0 & 1 & 1 & 0 & 0 & 0 & 1 & 0 & 0 & 1 \\
1 & 0 & 0 & 0 & 1 & 1 & 0 & 1 & 0 & 0 \\
0 & 0 & 0 & 1 & 0 & 1 & 0 & 1 & 1 & 0 \\
0 & 1 & 0 & 1 & 0 & 0 & 1 & 0 & 0 & 1 \\
1 & 0 & 1 & 0 & 1 & 0 & 0 & 0 & 1 & 0
\end{array}\right] \quad \begin{gathered}
\uparrow-K \\
\downarrow
\end{gathered}
$$

The output bits of the LDPC or TC channel encoders are then passed to the space-time block encoder of Figure 1. In our system, we employed Alamouti's $\mathrm{G}_{\mathbf{2}}$ space-time block code since it was shown in [3] that from the set of schemes investigated, the best performance was achieved by concatenating the space-time block code $\mathbf{G}_{2}$ with channel codes. The G2 space-time block code is associated with a twin-transmitter-based scheme, whose generator matrix is defined as follows:

$$
\mathbf{G}_{2}=\left(\begin{array}{cc}
x_{1} & x_{2} \\
-\bar{x}_{2} & \bar{x}_{1}
\end{array}\right) .
$$

The output of the space-time encoder is then OFDM modulated with the aid of the Inverse Fast Fourier Transform (IFFT) blocks of 


\begin{tabular}{|c|c|c|}
\hline Code & Code Rate & Puncturing Pattem \\
\hline $\mathrm{TC}(2,1,4)$ & 0.50 & 10,01 \\
\hline $\mathrm{TC}(2,1,4)$ & 0.67 & 1000,0001 \\
\hline $\mathrm{TC}(2,1,4)$ & 0.75 & 100000,000001 \\
\hline $\mathrm{TC}(2,1,4)$ & 0.83 & 1000000000,0000000001 \\
\hline
\end{tabular}

Table 2: Puncturing Pattern for $(2,1,4)$ TC Code [11].

Figure 1 and transmitted by the corresponding antenna. The number of transmit antennas is fixed to two, while the number of receive antennas constituted a design parameter."Dispersive wideband channels were considered and the associated channels' impulse response will be discussed at a later stage.

At the receiver, the signal of each receive antenna is OFDM demodulated. The demodulated signals of the receiver antennas are then fed to the space-time block decoder of Figure 1. The space-time decoders apply the Logarithmic Maximum A-Posteriori (Log-MAP) decoding algorithm [3] for providing soft outputs for the channel decoders. The LDPC code can be decoded by the so-called sum-product algorithm [8] or by belief propogation [9]. By contrast, the TC code is decoded with the aid of the Log-MAP algorithm [3].

\section{PERFORMANCE}

In this section, we provide our simulation results for the various spacetime coded QPSK modulated OFDM schemes [3] concatenated with TC and LDPC coding, while using 128 subcarriers. Each OFDM symbol has a duration of $160 \mu s$ and a cylic prefix of $40 \mu s$ duration. In these simulations, the Jake model was adapted for modelling the fading channels [10]. Again, we assume an equal-power two-path Channel Impulse Response (CIR), where the CIR taps are separated by a delay spread of $5 \mu \mathrm{s}$. The maximum Doppler frequency was 200 $\mathrm{Hz}$. All multipath components undergo independent Rayleigh fading and the receiver has a perfect knowledge of the CIR.

\subsection{Effect of various coding rates}

Let us now compare the achievable performance of the two channel coding schemes at different coding rates. As mentioned in Section 2 , the code rate $R$ of LDPC codes can be calculated as $R=K / N$, where $K$ is the difference between the number of columns and rows of the parity check matrix, and $N$ is the number of columns of the matrix determining the block length of the code. Different LDPC code rates can be readily created by adjusting the value of $K$ and $N$.

On the other hand, for TC we have to specify the correct puncturing pattern in order to produce the required code rates. The puncturing pattern used in our simulation is shown in Table 2, which is based on the approach proposed by Acikel $e t$ al. in [11]. Clearly, it is more straightforward to produce arbitrary code rates for LDPC codes compared to TC's.

The results of our simulations using QPSK and 16-QAM are shown in Figures 2 and 3 in terms of the achievable BER and FER, respectively. We can see from these figures that the LDPC code has almost the same BER performance as the TC and slightly outperforms TC in terms of the achievable FER, as the coding rate increases. From Figure 2 we can derive the effective Bits per Symbol (BPS) versus $\mathrm{E}_{b} / \mathrm{N}_{0}$ performance at $\mathrm{BER}=10^{-6}$. The corresponding results are shown in Figure 5. We can see that the performance of the LDPC code surpasses that of TCs, when the effective BPS throughput becomes higher than approximately 1.5 in conjunction with QPSK and 3.2 for 16-QAM, respectively. From Figure 3 we can also extract a

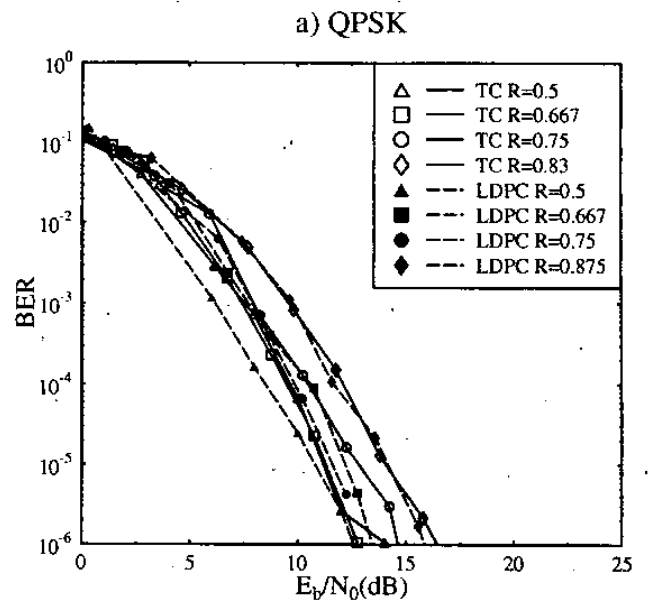

b) 16QAM

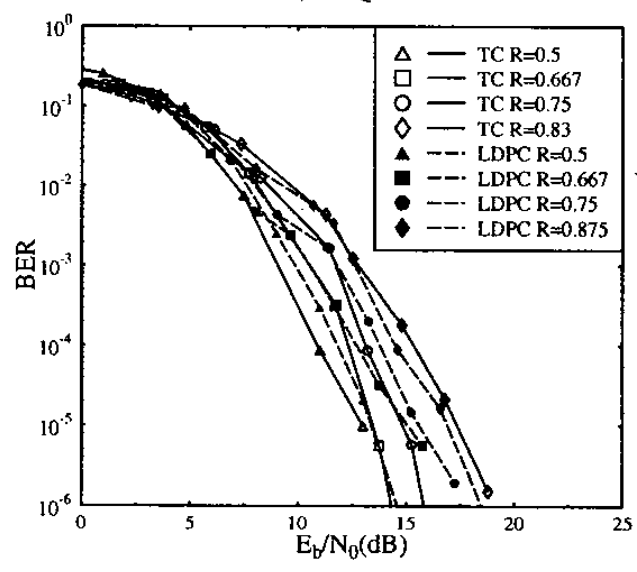

Figure 2: BER versus $E_{b} / N_{0}$ using TC and LDPC codes having various coding rates using $\mathbf{G}_{2}$ space-time block code (STBC) OFDM and one receiver antenna for a) QPSK and b) 16-QAM. The framelength of all schemes was 512 bits and a channel having a CIR characterised by two equal-power rays separated by a delay spread of $5 \mu \mathrm{s}$. The maximum Doppler frequency was $200 \mathrm{~Hz}$

plot of the $E_{b} / N_{o}$ values required for maintaining $F E R=10^{-3}$ versus the code rate. The derived plot is featured in Figure 4. This plot shows that LDPC codes require lower values of $E_{b} / N_{0}$ for reaching an FER value of $10^{-3}$ compared to TCs.

\subsection{Effect of the code length}

The input block length of the codes was also varied for the sake of investigating the effects of various coding delays on the performance of the system. In these investigations the number of iterations used 


\section{a) QPSK}

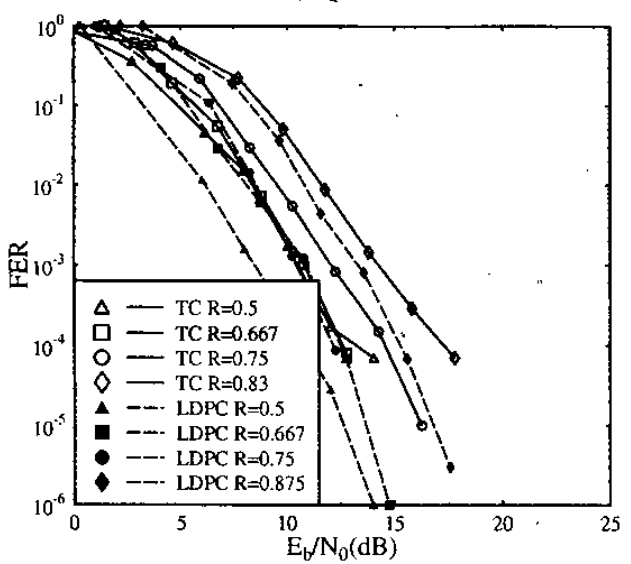

b) 16QAM

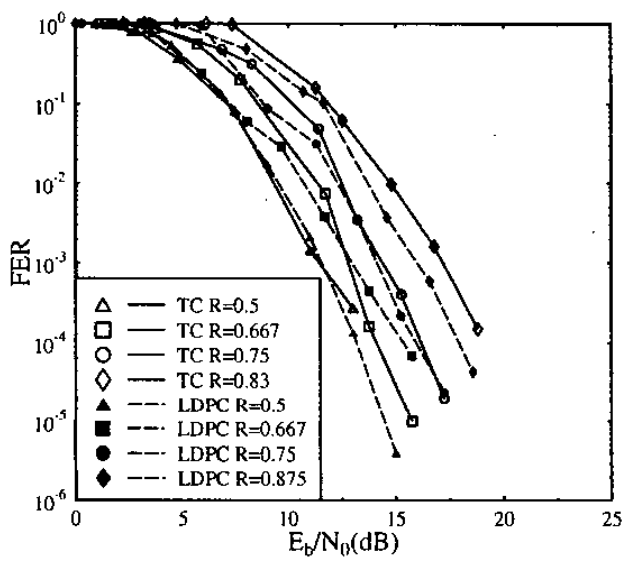

Figure 3: FER versus $E_{b} / N_{0}$ using TC and $L D P C$ with various coding rates using $\mathrm{G}_{2}$ STBC OFDM and one receiver antenna for a) QPSK and b) 16-QAM. The framelength of all schemes was 512 bits and a channel having a CIR characterised by two equal-power rays separated by a delay spread of $5 \mu \mathrm{s}$. The maximum Doppler frequency was $200 \mathrm{~Hz}$

by both the TC and LDPC codes was fixed to eight, the modulation scheme used was QPSK, and the code rate was 0.5 for both the TC and LDPC codes. In Figure 6 we can see that the FER of LDPC code becomes lower than that of the TC code, when we increase the input block length.

\subsection{Effects of the number of iterations}

In Figure $7(a)$, we characterise the achievable coding gain of both LDPCs and TCs, when different number of iterations are used. The achievable coding gain was defined here as the $\mathrm{E}_{b} / \mathrm{N}_{0}$ difference -

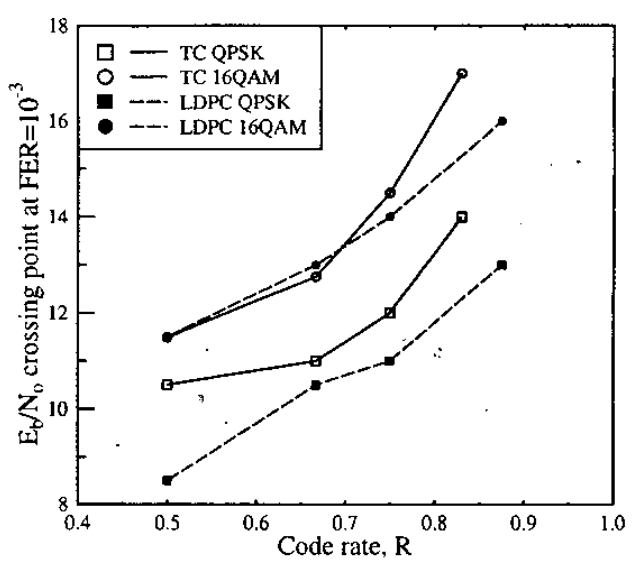

Figure 4: $E_{b} / N_{0}$ crossing point at $F E R=10^{-3}$ versus the coding rate for TC and LDPC coding using the STBC $G_{2}$ and one receiver antenna in a QPSK and 16-QAM modulated OFDM system. A channel having a CIR characterised by two equal-power rays separated by a delay spread of $5 \mu \mathrm{s}$ was used. The maximum Doppler frequency was $200 \mathrm{~Hz}$.

expressed in decibels $(\mathrm{dB})$ at $\mathrm{FER}=10^{-3}-$ between the proposed schemes and the uncoded single-transmitter, single-receiver system having the same effective throughput. In the case of Figure 7(a) the TC and LDPC codeword length has been fixed at 512 bits, the modulation scheme used is QPSK and the code rate is fixed at 0.5 . We can see from the figure that for a single receiver antenna based system the LDPC code performs less well at a low number of iterations, although its performance eventually reaches that of the $\mathrm{TC}$ after about 12 iterations. It also has to be mentioned that an LDPC iteration is typically less complex than a TC iteration, as it will be shown in quantitative terms in Section 4 and argued with reference to Figure 7(b).

\section{DECODING COMPLEXITY}

$\mathbf{T C}(\mathbf{n}, \mathbf{k}, \mathbf{K})$ Complexity: Let us now consider the decoding complexity of the TC $(2,1,4)$ scheme utilising the binary Log-MAP decoder [3]. Briefly, the a posteriori probability of a binary information bit $u_{t}, t \in\{0, \ldots, N-1\}$, given the received coded bit sequence of $\mathbf{y}=\left\{y_{0}, \ldots, y_{N n-1}\right\}$, where $N$ is the number of $n$-bit coded symbols, can be computed as follows [3]:

$$
\operatorname{Pr}\left\{u_{t}= \pm 1 \mid \mathbf{y}\right\}=\sum_{\substack{(s, s) \rightarrow \vec{t} \\ u_{t}= \pm 1}} \beta_{t}(s) \cdot \alpha_{t-1}(\grave{s}) \cdot \gamma_{t}(\grave{s}, s),
$$

where $(\grave{s}, s) \Rightarrow u_{t}= \pm 1$ indicates the specific set of trellis transitions emerging from the previous trellis state $S_{t-1}=\grave{s}$ to the present state $S_{t}=s$ that can be encountered, when the information bit is $u_{t}= \pm 1$. Furthermore, $\alpha_{t}(s), \beta_{t-1}(\grave{s})$ and $\gamma_{t}(\grave{s}, s)$ are the forward recursion, backward recursion and branch transition metric, respectively [3]. The Log Likelihood Ratio (LLR) of $\operatorname{Pr}\left\{u_{t}= \pm 1 \mid y\right\}$ can 


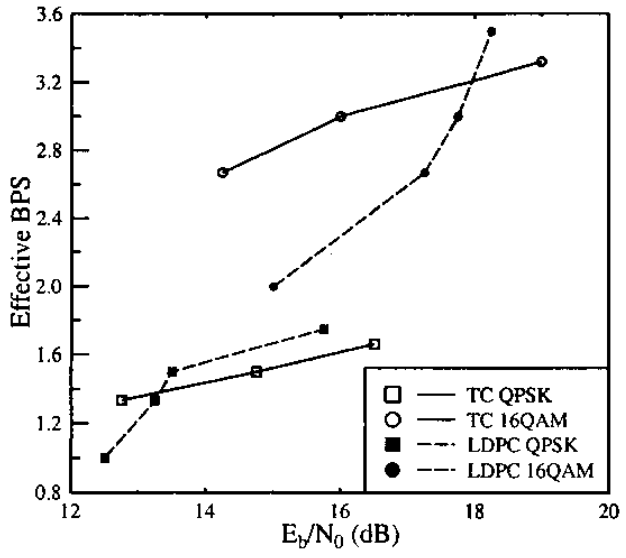

Figure 5: Effective BPS throughput versus $\mathrm{E}_{b} / \mathrm{N}_{0}$ using TC and LDPC codes having various coding rates using the STBC $\mathbf{G}_{2}$ and one receiver antenna in an OFDM system at $B E R=10^{-6}$. A channel having a CIR characterised by two equal-power rays separated by a delay spread of $5 \mu$ s was used. The maximum Doppler frequency was $200 \mathrm{~Hz}$.

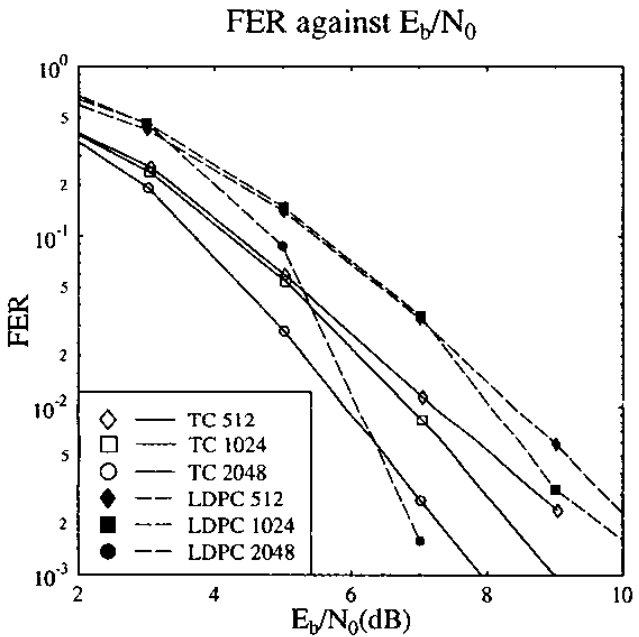

Figure 6: FER versus $E_{b} / N_{0}$ in conjunction with different input block lengths for both the half-rate TC and LDPC codes employing eight iterations concatenated with the $S T B C \mathrm{G}_{2}$ using one receiver and a 128-subcarrier QPSK modulated OFDM modem. The CIR was characterised by two equal-power rays separated by a delay spread of $5 \mu \mathrm{s}$. The maximum Doppler frequency was $200 \mathrm{~Hz}$. The length of the turbo interleaver was half of the channel interleaver, which resulted in the same memory or delay.

be computed as [3]:

$$
L\left(u_{t} \mid \mathbf{y}\right)=\ln \left(\frac{\operatorname{Pr}\left\{u_{t}=+1 \mid \mathbf{y}\right\}}{\operatorname{Pr}\left\{u_{t}=-1 \mid \mathbf{y}\right\}}\right) .
$$

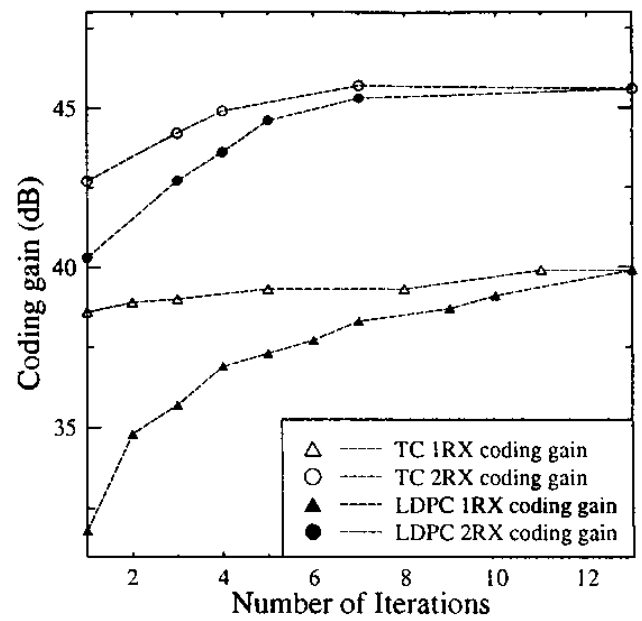

(a)

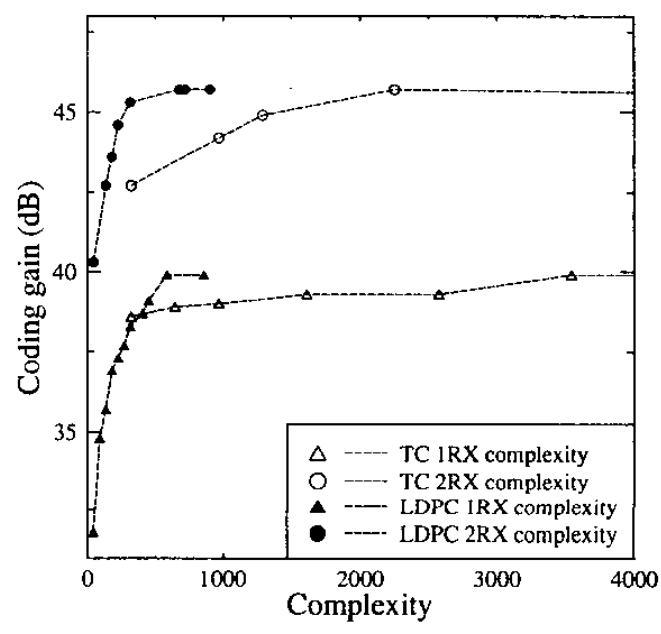

(b)

Figure 7: Coding gain extracted from the FER versus $\mathrm{E}_{b} / \mathrm{N}_{0}$ curves at FER $=10^{-3}$ as a function of (a) the number of iterations and (b) versus estimated complexity for the half-rate TC and LDPC coded STBC $\mathbf{G}_{2}$ OFDM scheme using one or two receiver antennas and a 128-subcarrier QPSK OFDM modem for transmission over a channel having a CIR characterised by two equal-power rays separated by a delay spread of $5 \mu \mathrm{s}$. The maximum Doppler frequency was $200 \mathrm{~Hz}$.

Explicitly, we have $\alpha_{t}(s)=\sum_{\text {all } \grave{s}} \gamma_{t}(\grave{s}, s) \cdot \alpha_{t-\mathrm{I}}(\grave{s}), \beta_{t-1}(\grave{s})=$ $\sum_{\text {all } s} \beta_{t}(s) \cdot \gamma_{t}(\grave{s}, s)$ and $\gamma_{t}(\grave{s}, s)=\Pi_{t, u_{t}} \cdot \eta_{t}(\grave{s}, s)$. Specifically, $\Pi_{t, u_{t}}$ is the a priori information regarding the information bit $u_{t}$ and $\eta_{t}(\grave{s}, s)=\exp \left(\frac{1}{2} \sum_{l=1}^{n} L\left(x_{n t+l} \mid \mathbf{y}\right) x_{n t+l}\right)$ where $x_{n t+1}, \ldots, x_{n t+n}$ are the legitimate transmitted coded bits corresponding to the infor- 
mation bit $u_{t}$ when a state transition from the previous trellis state $\grave{s}$ to the present state $s$ occurred. Furthermore, $L\left(x_{n t+l} \mid \mathbf{y}\right)$ is the LLR of $x_{n t+i}$ given the received coded bit sequence $\mathrm{y}$, which is obtained at the demodulator.

Let us now determine the complexity of the MAP decoder associated with evaluating $\operatorname{Pr}\left\{u_{t}=+1 \mid y\right\}$ based on Equation 3. In order to compute the term $\eta_{t}(\grave{s}, s)$ given $L\left(x_{n t+l} \mid \mathbf{y}\right)$, we need $n+1$ multiplications and $n-1$ additions. Note that the exponential function $\eta_{t}\left(\grave{s}_{1} s\right)$ is cancelled out when the Log-MAP decoder is employed When evaluating the term $\gamma_{t}(\grave{s}, s)$ using $\Pi_{t, a}$ and $\eta_{t}$, we need only one multiplication since there is only one trellis transition emerging from the previous trellis state $\grave{s}$ to the present state $s$ that can be encountered, when the information bit is $u_{t}=+1$. As for $\alpha_{t}$ and $\beta_{t}$, each term requires $S$ number of multiplications and $S-1$ number of additions, where we have $S=2^{K-1}$ and $K$ is the constraint length of the code. Finally, the evaluation of the term $\operatorname{Pr}\left\{u_{t}=+1 \mid y\right\}$ requires $2 S$ number of multiplications and $S-1$ number of additions. Therefore, a total of $(n+1)+1+4 S=4 S+n+2$ number of multiplications and $(n-1)+3(S-1)=3 S+n-4$ number of additions are required for computing $\operatorname{Pr}\left\{u_{t}=+1 \mid y\right\}$. However, we also have to calculate $\operatorname{Pr}\left\{u_{t}=-1 \mid \mathbf{y}\right\}$ and compute the corresponding ratio in order to evaluate the LLR of Equation 4. Therefore, a total of $2(4 S+n+2)+1=8 S+2 n+5$ number of multiplications/divisions and $2(3 S+n-4)$ number of additions are required by one MAP decoder for decoding the binary information bit $u_{t}$. Since two MAP decoders are required in the TC decoder, which performs $T$ number of turbo iterations for decoding a block of $N$ information bits, the estimated complexity of the TC scheme per information bit per iteration is $2(8 S+2 n+5)$ multiplications/divisions plus $4(3 S+n-4)$ number of additions.

When the log-MAP decoder is employed for the sake of reducing the computational complexity imposed, the multiplication/division operation is substituted by addition/subtraction in the logarithmic domain. Furthermore, the addition operation is substituted by the Jacobian sum operations [12] when it is carried out in the logarithmic domain. More specifically, each Jacobian sum consists of an addition, a subtraction, a table look-up and a maximum evaluation operation [12]. However, we can ignore the table lookup and max operations due to their comparably low complexity. As a result, the total complexity of the TC scheme per information bit per iteration in terms of additions and subtractions is:

$$
\begin{aligned}
\operatorname{comp}\{T C(n, 1, K)\}= & 2(8 S+2 n+5)+8(3 S+n-4) \\
= & 40\left(2^{K-1}\right)+12 n-22 \\
& \text { where } S=2^{K-1} .
\end{aligned}
$$

LDPC $(j, k)$ Complexity: The decoding complexity per information bit per iteration of LDPC codes in conjunction with a parity check matrix having a column weight of $j$ and a row weight of $k$ can be approximated in terms of additions and subtractions when operating in the logarithmic domain [13] as:

$$
\operatorname{comp}\{L D P C\}=(4 k+j) j
$$

From Equations 5 and 6, we can see that the complexity of decoding one bit in one iteration is lower in LDPC codes compared to TC codes. For the systems used in our simulations, the complexity of a TC $(2,1,4)$ code is calculated to be 322 using Equation 5. However, from Equation 6, the complexity of LDPC is just 45 since the column weight, $k$ and row weight, $j$ used were 3 . As an example, a single $\mathrm{TC}(2,1,4)$ iteration has a similar complexity to about 7 LDPC iterations for the same code. Hence Figure 7 (b) shows the coding gain achievable versus the estimated complexity of the $\mathrm{TC}(2,1,4)$ and LDPC codes for the same codelength.

\section{CONCLUSION}

In this paper we have presented a performance comparison of concatenating $\mathrm{G}_{2}$ space-time block coded OFDM schemes with both TC and LDPC. It has been shown that the two channel coding schemes perform fairly similarly. However, LDPC codes tend to have a lower decoding complexity compared to TCs.

\section{REFERENCES}

[1] L. Hanzo, W. Webb and T. Keller, Single and Multi-carrier Quadrature Amplitude Modulation. Wessex, England: John Wiley \& Sons Ltd., 2000.

[2] D. Agrawai, V. Tarokh, A. Naguib, and N. Seshadri, "Spacetime Coded OFDM for High Data-rate Wireless Communication Over Wideband Channels," in Proceedings of IEEE VTC 1998, (Ottawa, Canada), pp. 2232-2236, 1998.

[3] L. Hanzo, T. H. Liew and B. L. Yeap, Turbo Coding, Turbo Equalisation and Space Time Coding for Transmission over Wireless channels. West Sussex, England: John Wiley and IEEE Press, 2002.

[4] R. G. Gallager, "Low Density Parity Check Codes," IRE Transactions on Information Theory, vol. IT-8, pp. 21-28, January 1962.

[5] H. Futaki and T. Ohtsuki, "Low-Density Parity-Check (LDPC) Coded OFDM Systems," in Proceedings of IEEE VTC Fall 2001, (Atlantic City, USA), pp. 82-86, 2001.

[6] B. Lu, X. Wang and K. R. Narayanan, "LDPC-Based SpaceTime Coded OFDM Systems Over Correlated Fading Channels: Performance Analysis and Receiver Design," IEEE Transactions on Communications, vol. 50, pp. 74-88, January 2002.

[7] S. M. Alamouti, "A Simple Transmit Diversity Technique for Wireless Communications," IEEE JSAC, pp. 145I-1458, October 1998.

[8] D. J. C. MacKay and R. M. Neal, "Near Shannon Limit Performance of Low Density Parity Check Codes," Electronics Letters, vol. 32, pp. 1645-1646, August 1996.

[9] L. Ping and W. K. Leung, "Decoding Low Density Parity Check Codes wih Finite Quantization Bits," IEEE Communications Letters, vol. 4, pp. 62-64, February 2000.

[10] W. C. Jakes, Microwave Mobile Communications. Piscataway, New Jersey, USA: IEEE Press, 1993.

[11] O. Acikel and W. Ryan, "Punctured turbo-codes for BPSK/QPSK channels," IEEE Transactions on Communications, vol. 47, pp. 1315-1323, September 1999.

[12] P. Robertson, E. Villebrun and P. Höher, "A Comparison of Optimal and Sub-Optimal MAP Decoding Algorithms Operating in the Log Domain," in Proceedings of IEEE International Conference on Communications, pp. 1009-1013, 1995.

[13] F. Guo and S. X. Ng and L. Hanzo, "LDPC assisted Block Coded Modulation for Transmission over Rayleigh Fading Channels ," in Proceedings of IEEE VTC 2003 Spring, (Jeju, Korea), 2003. 